\title{
Optimierung von Epoxidharzsystemen für PE-Rohrinnenbeschichtungen als Sauerstoffsperrschicht
}

\author{
Eckhart Kornejew, Harald Goering, Michael Herzog*
}

\section{Zusammenfassung}

Gegenwärtig haben bei Fußbodenheizungen Kunststoffrohre aus Polyethylen (PE) oder Polypropylen (PP) Stahlund Kupferrohre fast vollständig ersetzt. Trotz der überragenden Gebrauchseigenschaften dieser Kunststoffrohre stellen die relativ hohe Sauerstoffpermeabilität und die geringere Langzeitstabilität ein Problem dar. Sauerstoff reagiert mit den Metallwerkstoffen in den Systemkomponenten, was zu einer beschleunigten Korrosion führt, und schädigt das PE durch eine Radikal-initiierte autokatalytische Kettenreaktion. Diese Reaktion führt zu einer Versprödung des PE bis hin zur Rissbildung. Dadurch wird die Sauerstoffpermeabilität des PE zusätzlich drastisch erhöht. Das kann eine nachträglich eingebrachte Sauerstoffbarriereschicht auf Epoxidharzbasis unterbinden.

Mit Hilfe der Differential Scanning Calorimetry (DSC) werden Untersuchungen zur Struktur-Eigenschaftskorrelation durchgeführt, um das Eigenschaftsprofil des Epoxidharzes zu optimieren. Variiert werden das Harz-HärterVerhältnis, die Zusammensetzung des Harz- und HärterSystems, einschließlich eines reaktiven Verdünners, sowie der eingesetzte Katalysator. Als Charakteristika werden die Glasübergangstemperatur $\mathrm{T}_{\mathrm{g}}$ des härtenden Systems und die bei der Härtung auftretende Reaktionsenthalpie $\Delta \mathrm{H}$ diskutiert.

\begin{abstract}
Presently, metal tubes in underfloor heating systems are nearly completely substituted by plastic tubes made of polyethylene (PE) or polypropylene (PP). In spite of the extraordinary properties of these plastic tubes in use, issues that remain are the relatively high oxygen permeability and the lower longtime durability compared to metal tubes. Oxygen tends to react with the metal components of the systems leading to an accelerated corrosion. The $P E$ is affected by a radically initiated auto-catalytic chain reaction. This leads to an embrittlement of the PE, and finally to the formation of micro-cracks and visual cracks. Thus, the oxygen permeability is additionally increased by a high rate. This mechanism may be prevented through an oxygen barrier layer applied into running systems.

Investigations by differential scanning calorimetry (DSC) were performed aiming at the elucidation of structure property relationships to optimize the application properties of the epoxy resins used in barrier layer production. Here, variations were made in the equivalent ratio of resin and hardener, the composition of the resin hardener system including a reactive diluent and a catalyst. Characteristic parameters, such as the glass transition temperature of the curing system and the reaction enthalpy liberated during the curing process, are discussed.
\end{abstract}

\section{Einführung}

Für Fußbodenheizungen werden seit den 60er Jahren Rohre aus Polyethylen (PE) eingesetzt. Inzwischen haben in diesem Bereich Kunststoffrohre fast vollständig Stahlrohre und auch Kupferrohre ersetzt. PE nimmt dabei wegen seines besonderen Eigenschaftsspektrums einen herausragenden Platz ein. Rohre aus diesem Material sind vergleichsweise sehr leicht und lassen eine vorteilhafte Rohrverbindung durch Schweißen und mit Hilfe von Steckmuffen oder Klemmfittings zu. Die Nachteile einer geringeren Tempe- raturbeständigkeit und Steifigkeit von PE gegenüber Metallen fallen beim Einsatz in Fußbodenheizungen kaum ins Gewicht.

Probleme bei der Anwendung von PE als Rohrmaterial für Fußbodenheizungen stellen dagegen die relativ hohe Sauerstoffpermeabilität und das "langsame Risswachstum" (Langer et al. 2012) dar. Obwohl die Permeabilität für Gase im Allgemeinen gegenüber anderen Kunststoffen bei PE relativ gering ist, kann Sauerstoff PE recht gut durchdringen und so in den Heizwasserkreislauf gelangen. Das wiederum kann zu Korrosionsschäden an den Metallbau- teilen der Heizungsanlage führen sowie die Rohre selbst schädigen. Neben der gezielten Herstellung von speziellen (wegen der enorm gewachsenen Möglichkeiten durch die rasante KatalyseEntwicklung) oder vernetzten PE-Typen können in der letzten Zeit Lackierungen, Schutzfolien oder Sperrschichten sauerstoffdichte Rohre nach DIN 4726 gewährleisten. Allerdings stellen sämtliche Verbindungselemente für die einzelnen Anlagenkomponenten und insbesondere das Ausgleichsgefäß Schwachstellen für eine Gaspermeation dar. Fußbodenheizungen gelten dabei als besonders gasdurchlässige Anla- 
gen, auch bei Berücksichtigung der die PE-Rohrschlange umhüllenden EstrichSchicht (Sittiho 2011). Das hauptsächliche Problem sind die Korrosion und Korrosionsprodukte durch Reaktion des Sauerstoffs mit den Eisenwerkstoffen in den Systemkomponenten. Weiterführende Literatur hat Sittiho (2011) zusammengestellt.

Der im System enthaltene und von außen eindringende Sauerstoff hat aber auch schädigenden Einfluss auf die PERohrwandungen. Sauerstoff bildet im PE Sauerstoffradikale, die zur Reaktion mit den Methyl- bzw. Methylengruppen führen und dabei Sauerstoffverbindungen bilden, z. B. Aldehyd-, Carboxyl-, Keto- oder Hydroxylgruppen. Durch die Bildung dieser Gruppen wird die Fähigkeit des so geschädigten PE zur Aufnahme und zur Permeation von Sauerstoff wesentlich erhöht. Diese chemischen Reaktionen wirken sich wiederum dramatisch auf die physikalische Struktur und damit auf die mechanischen Eigenschaften aus.

\section{Chemie der Alterung von PE}

Der thermo-oxidative Abbau von PE ist eine Radikal-initiierte autokatalytische Kettenreaktion (Gugumus 1996a, 1996b, 1996c). Diese Reaktion ist zwar zu Beginn vergleichsweise langsam, beschleunigt sich jedoch mit zunehmender Konzentration der entstehenden Hydroperoxide. Die Reaktion verläuft in drei Schritten: Ketteninitiierung, Kettenfortschritt und Kettenabbruch (Kriston 2010):

\section{Ketteninitiierung:}

$\mathrm{R}-\mathrm{R} \rightarrow \mathrm{R}^{\circ}+\mathrm{R}^{\circ}$

\section{Kettenfortschritt:}

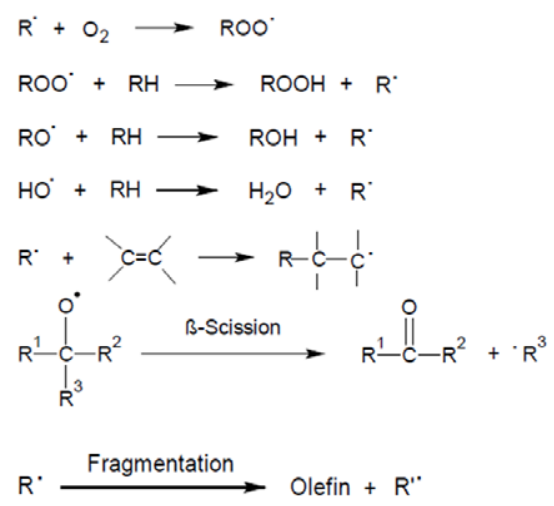

Kettenverzweigung:

$\mathrm{ROOH} \longrightarrow \mathrm{RO}^{*}+{ }^{\circ} \mathrm{OH}$
$2 \mathrm{ROOH} \longrightarrow \mathrm{RO}^{\circ}+\mathrm{ROO}^{\circ}+\mathrm{H}_{2} \mathrm{O}$

Kettenabbruch:

$\mathrm{R}^{*}+\mathrm{ROO}^{*} \longrightarrow \mathrm{ROOR}$

$\mathrm{R}^{*}+\mathrm{R}^{\cdot} \longrightarrow \mathrm{R}-\mathrm{R}$

$\mathrm{RO}^{*}+\mathrm{R}^{*} \longrightarrow \mathrm{R}-\mathrm{O}-\mathrm{R}$

$2 \mathrm{R}^{*} \quad \longrightarrow \quad \mathrm{RH}+$ Olefin

Das primäre Alkylradikal R` kann durch Radikalinitiatoren (aus der Herstellung des PE), durch Katalysatorreste, durch Wärme, mechanische Kräfte (Scherung), Verunreinigungen, energiereiche Strahlung oder Additive gebildet werden. Die Alkylradikale reagieren mit molekularem Sauerstoff praktisch ohne Aktivierungsenergie und bilden Peroxyradikale:

$\mathrm{R}^{\cdot}+\mathrm{O}_{2} \longrightarrow \mathrm{ROO}^{\circ}$

Die Peroxyradikale bilden ihrerseits Hydroperoxide durch Abspaltung eines Wasserstoffatoms von der Polymerkette, wodurch eine $\mathrm{C}-\mathrm{H}$-Bindung gespalten werden muss:

$\mathrm{ROO}^{*}+\mathrm{RH} \longrightarrow \mathrm{ROOH}+\mathrm{R}^{*}$

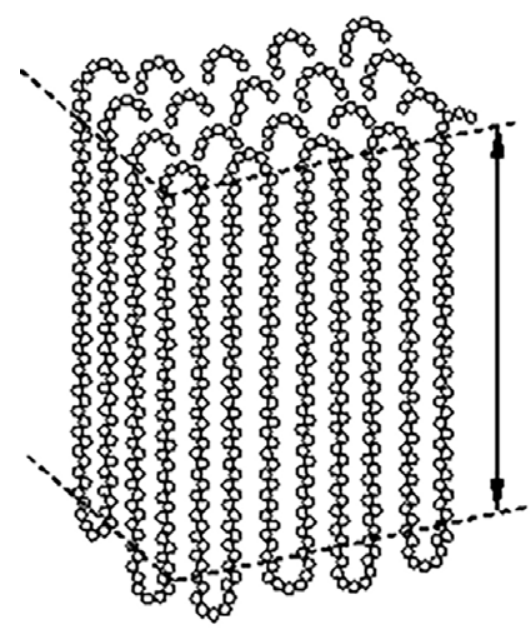

a)
Diese Reaktion ist der geschwindigkeitsbestimmende Schritt bei der thermisch-oxidativen Alterung der Polyolefine (Autoxidation), da diese Reaktion Aktivierungsenergie erfordert. Die Peroxyradikale können die Polyolefine weiter oxidieren, wobei dann Carbonsäuregruppen, Carbonsäureester oder Epoxide entstehen.

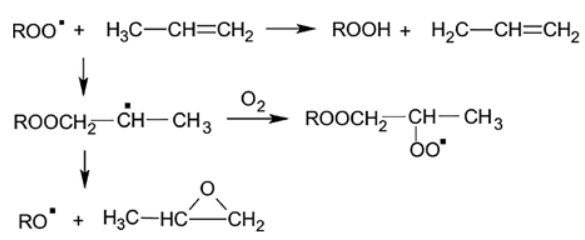

Die Geschwindigkeit der Kettenspaltung steigt mit der Temperatur.

Weitere Reaktionen werden durch Metallionen initiiert bzw. katalysiert:

$\mathrm{M}^{\mathrm{n}+}+\mathrm{ROOH} \longrightarrow \mathrm{M}^{(\mathrm{n}+1)+}+\mathrm{RO}^{\circ}+\mathrm{HO}^{-}$
$\mathrm{M}^{(\mathrm{n}+1)+}+\mathrm{ROOH} \longrightarrow \mathrm{M}^{\mathrm{n}+}+\mathrm{ROO}^{\circ}+\mathrm{H}^{+}$

Die Oxy- und Hydroxylradikale, die bei der Zersetzung der Hydroperoxide gebildet werden, sind wesentlich reaktiver als die Peroxyradikale und führen zu einer Verzweigung der Kettenreaktion, d. h. zu einer automatischen Beschleunigung der Reaktion und damit des Abbauprozesses. Die $\beta$-Spaltung der Oxy-Makroradikale führt zu Carbonylgruppen und weiteren freien Radikalen.

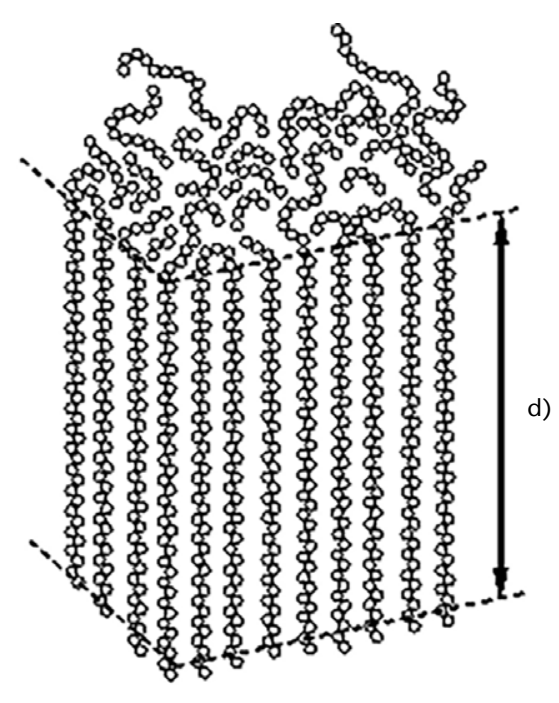

b) 
Zur Verhinderung oder zur Reduzierung der Alterungsprozesse werden den Polyolefinen Alterungsschutzmittel (auch Stabilisatoren oder Korrosionsschutzmittel) zugesetzt. Bei diesen handelt es sich um chemische Verbindungen, die in der Lage sind, freie Radikale oder instabile Zwischenprodukte "einzufangen“ und sie in stabile Produkte umzuwandeln, siehe Literaturangaben bei Kriston (2010). Das Abfangen der primären Makroradikale würde die Autoxidation unterbinden. Da die Reaktionsgeschwindigkeit des molekularen Sauerstoffs sehr hoch ist, kann die Radikalbildung nur sehr schwer unterbunden werden. Es gibt jedoch einige Verbindungen, die die Radikalbildung zumindest für eine gewisse Zeit wirksam verhindern, indem sie stabile Verbindungen bilden. $\mathrm{Zu}$ diesen geeigneten Verbindungen zählen insbesondere phenolische Antioxidantien, d. h. Phenole mit Substituenten in der 2,4,6-Position, wobei die Substituenten in der Regel verzweigte Alkylgruppen sind, z. B. tertButyl- oder Isopropylgruppen. Weitere Gruppen sind sterisch gehinderte aromatische Amine, sterisch gehinderte sekundäre Amine oder phosphororganische Verbindungen, in selteneren Fällen auch organische Schwefelverbindungen. Alle Stabilisatoren haben jedoch den Nachteil, dass sie aus dem PE an die Oberfläche migrieren und dann ausgewaschen werden können (Ojeda et al. 2011, Viebke \& Gedde 1997).

\section{Morphologie von PE}

Neben dem molekularen Aufbau der Ketten hat die gegenseitige Anordnung der Ketten im Polymermaterial, d. h. die Morphologie, entscheidenden Einfluss auf das Verhalten des Polymers. Diese ist sowohl vom molekularen Aufbau der Makromoleküle als auch von deren Molmasse (Kettenlänge) abhängig. Bei den Faltkristallen treten neben der regulär gefalteten Anordnung mit benachbartem Wiedereintritt der Ketten auch lockere Schleifen mit statistischem Wiedereintritt der Ketten (Switchboard-Modell) und lose hervorstehende Kettenenden auf.

Für die Beschreibung der entstehenden Ordnungsstruktur werden in der

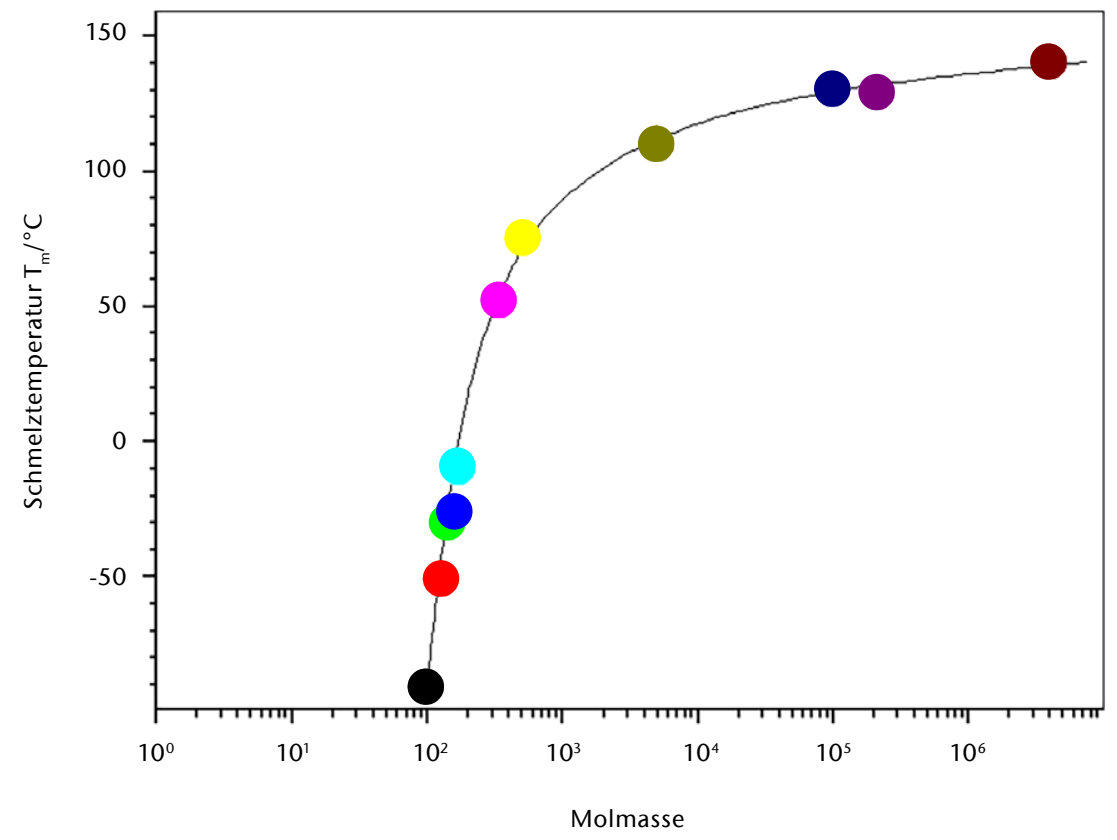

Abb. 2) Schmelztemperatur $T_{m}$ in Abhängigkeit von der Molmasse.
Literatur verschiedene Modelle gemäß Abb. 1 diskutiert. Die Abb. 1a zeigt das Modell einer regulär gefalteten Anordnung mit benachbartem Wiedereintritt der Ketten (Bildung bevorzugt aus einer Lösung) und Abb. 1b das Switchboard-Modell mit statistischem Wiedereintritt der Ketten (bevorzugt aus der Schmelze gebildet). Diese spezielle morphologische Struktur bestimmt das jeweilige Eigenschaftsspektrum des PE, d. h. es gibt nicht das eine Eigenschaftsprofil für $\mathrm{PE}$, sondern eine Vielfalt von Varianten.

Neben den Parametern des Herstellungsverfahrens, die sowohl die Verzweigungsstruktur als auch die Molmasse und ihre Verteilung festlegen, hat auch die thermische Geschichte des PE-Materials entscheidenden Einfluss auf die Morphologie und damit auf die Eigenschaften. Dabei spielt die Kettenlänge, d. h. die Molmasse des
PE, eine maßgebliche Rolle für die ausgebildete Morphologie. So korreliert die Schmelztemperatur $\mathrm{T}_{\mathrm{m}}$ über die Lamellendicke der Faltkristalle mit der Molmasse, d. h., durch die Kettenspaltung beim thermo-oxidativen Abbau werden die Eigenschaften des PE maßgeblich verändert.

In Abb. 2 werden Beispiele von Literaturwerten für $T_{m}$ in Abhängigkeit von der Molmasse (Kettenlänge bzw. Zahl der $\mathrm{CH}_{2}$-Sequenzen einer Molekülkette) gezeigt (Franck \& Biederbick 1990). Die gespaltenen und damit immer kürzer werdenden Ketten können sich besser umlagern und somit eine weitere Kristallisation des PE verursachen. Das bedeutet aber auch, dass mit kürzer werdenden Ketten die Kristallite kleiner werden (siehe $T_{m}$ ).

Hauptsächlich findet die Kettenspaltung in den zwischenkristallinen Be-

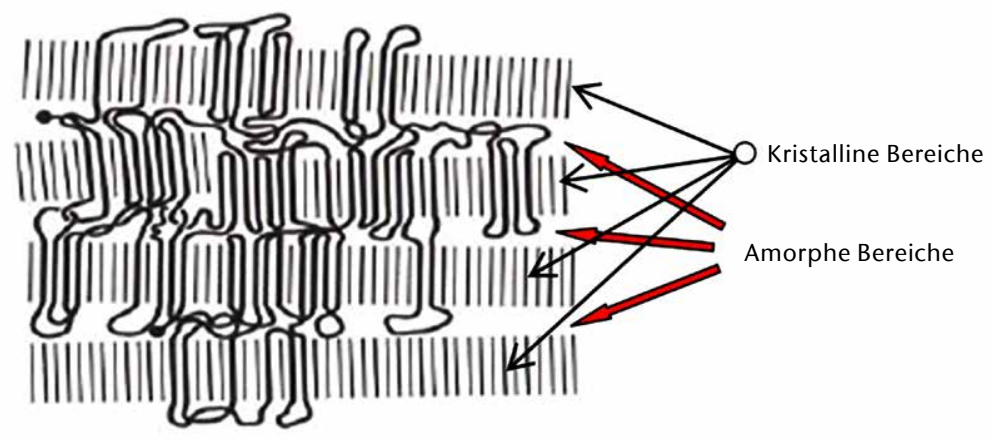

Abb. 3) Faltungsblöcke mit durchlaufenden Ketten in den amorphen Zwischenschichten 
reichen statt (wegen der größeren molekularen Beweglichkeit und der weniger kooperativen zwischenmolekularen Wechselwirkungen sowie der leicht „verspannten“ Valenzwinkel). Damit führt eine Kettenspaltung sowohl zu einer drastischen Verringerung der mechanischen Eigenschaften des PE als auch zur Veränderung von Transporteigenschaften, z. B. der Gaspermeabilität.

Das Strukturmodell des partiell kristallinen PE (Weltschev 2010) in Abb. 3 soll veranschaulichen, dass durch oxidative Kettenspaltung in den amorphen Bereichen der mechanische Zusammenhalt, der vor allem von durchlaufenden Ketten und Verschlaufungen gegeben wird, schnell verloren geht (begleitet von einer Nachkristallisation und damit einer Versprödung und schließlich Rissbildung).

Für Alterungsprozesse im PE spielen neben dem Vorhandensein von Sauerstoff auch Verbindungen eine wichtige Rolle, die im PE durch die Herstellungsund Verarbeitungsprozesse oder durch Wechselwirkung mit der Umgebung während des Gebrauchs eingebracht wurden. So wurde z. B. von Mitroka et al. (2013) der Einfluss von chloriertem Wasser auf das Langzeitverhalten von PE-Rohren untersucht.

\section{Problemstellung}

Auch wenn in den letzten Jahren Rohrsysteme auf eine Lebensdauer von 50 a ausgelegt werden, diskutiert man gegenwärtig über eine angestrebte Lebensdauer von 100 a (Vogt et al. 2008). Eine kritische Einschätzung geben neben einer Fülle von Literaturhinweisen Whelton \& Dietrich (2009). Die bisher vorgestellten Methoden der

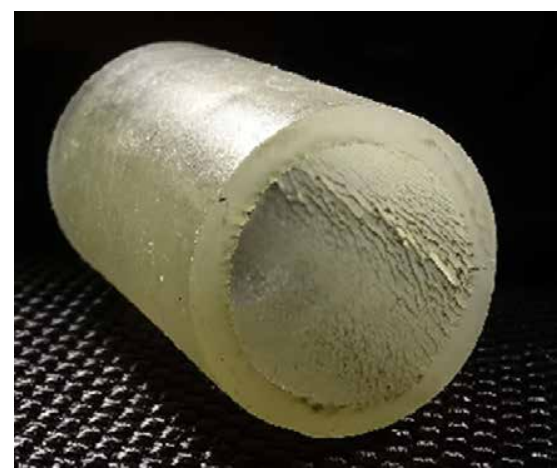

Abb. 4) PE-Rohr nach 34 Jahren Gebrauch in einer Fußbodenheizung. (Durchmesser $17 \mathrm{~mm}$, Wandstärke $2 \mathrm{~mm}$ )

Tab.1: Untersuchte Proben

\begin{tabular}{|c|c|c|c|c|}
\hline Bezeichnung & Es & Et & $\mathrm{DA}_{1}$ oder $\mathrm{DA}_{1+2}$ & Index \\
\hline $\mathrm{DA}_{1} 100$ & 100 & 0 & $\mathrm{DA}_{1} 100$ & 100 \\
\hline $\mathrm{DA}_{1+2} 100$ & 100 & 0 & $\mathrm{DA}_{1+2} 100$ & 100 \\
\hline $\mathrm{DA}_{1+2} 85$ & 100 & 0 & $\mathrm{DA}_{1+2} 85$ & 85 \\
\hline $\mathrm{DA}_{1+2} 8570 \mathrm{G}$ & 100 & 0 & $\mathrm{DA}_{1+2} 85$ & 85 \\
\hline $\mathrm{DA}_{1+2} 95$ & 100 & 0 & $\mathrm{DA}_{1+2} 95$ & 95 \\
\hline $\mathrm{DA}_{1+2} 90$ & 100 & 0 & $\mathrm{DA}_{1+2} 90$ & 90 \\
\hline $\mathrm{DA}_{1+2} 80$ & 100 & 0 & $\mathrm{DA}_{1+2} 80$ & 80 \\
\hline DA,95 & 100 & 0 & $\mathrm{DA}_{1} 95$ & 95 \\
\hline $\mathrm{DA}_{1} 90$ & 100 & 0 & $\mathrm{DA}_{1} 90$ & 90 \\
\hline $\mathrm{DA}_{1} 80$ & 100 & 0 & $\mathrm{DA}_{1} 80$ & 80 \\
\hline $\mathrm{DA}_{1} 95$ & 100 & 0 & $\mathrm{DA}_{1} 95$ & 95 \\
\hline $\mathrm{DA}_{1} 90-90 / 10$ & 90 & 10 & $\mathrm{DA}_{1} 90-90 / 10$ & 90 \\
\hline $\mathrm{DA}_{1} 90-80 / 20$ & 80 & 20 & $\mathrm{DA}_{1} 90-80 / 20$ & 90 \\
\hline $\mathrm{DA}_{1} 80-90 / 10$ & 90 & 10 & $\mathrm{DA}_{1} 80-90 / 10$ & 80 \\
\hline $\mathrm{DA}_{1} 90-95 / 5$ & 95 & 5 & $\mathrm{DA}_{1} 90-95 / 5$ & 90 \\
\hline $\mathrm{DA}_{1} 90+10$ & 100 & 0 & $\mathrm{DA}_{1} 90+10$ & 100 \\
\hline $\mathrm{DA}_{1} 100 / 90-10$ & 90 & 10 & $\mathrm{DA}_{1} 100 / 90-10$ & 100 \\
\hline
\end{tabular}

beschleunigten Bestimmung der Lebensdauer von PE in der industriellen Anwendung bedürfen weiterhin der kritischen Diskussion (Schröder et al. 2008, Robertson 2013).

Wie dramatisch die Schädigung eines PE-Rohres nach einem 34-jährigen Gebrauch in einer Fußbodenheizung sein kann, zeigt das Foto der Fa. TGA Rohrinnensanierung AG, Fürth, in Abb. 4.

Auf dem Foto ist zu erkennen, dass neben dem Verlust der mechanischen Festigkeit auch solche Eigenschaften wie die Permeabilität für Sauerstoff sehr stark verändert werden. Das Rohr verliert seine Barriereeigenschaften total, bis hin zur Wasserdurchlässigkeit. Eine in der Praxis bewährte Methode zur Beseitigung der Folgen solcher Schädigung ist die Rohrinnensanierung, bei der in das geschädigte Rohr eine Kunststoff-Barriereschicht eingebracht wird. Sie verhindert nach einer Entwicklung der Fa. TGA Rohrinnensa- nierung $A G$, Fürth, weitestgehend das Eindringen von Sauerstoff durch das Rohr in den Heizkreislauf sowie eine Migration der im PE enthaltenen Stabilisatoren und die Diffusion von Sauerstoff aus dem Heizwasser in die Rohrwandung, d. h., die Folgen der vor allem oxidativen Schädigung des PE werden kompensiert und eine weitere Schädigung durch Ausschluss von Sauerstoff erheblich reduziert. Auch kann mit dieser Rohrinnenbeschichtung ein erhöhter Korrosionsschutz für die metallischen Komponenten der bestehenden Heizanlage erreicht werden. Das Sanierungsverfahren lässt sich direkt auf Fußbodenheizungen mit anderen Kunststoffrohrmaterialien wie Polypropylen oder Polyisobutylen übertragen.

Neben der Wirkung als Sauerstoffbarriere muss das von innen eingebrachte Material eine Reihe von Eigenschaften aufweisen, die dem praktischen Verfahren geschuldet sind, wie Topfzeit, 
Gelzeit, Härtungszeit, Viskosität u. a. Dazu wurde in einem F+E-Vorhaben der TH Wildau mit der Fa. TGA Rohrinnensanierung AG, Fürth, ein Epoxidharzsystem mit kommerziellen Komponenten entwickelt, das die in der DIN 4726 geforderte Sauerstoffdichtheit um mehr als eine Zehnerpotenz übertrifft (Prüfbericht 2014). Die Optimierung des Eigenschaftsspektrums dieses Epoxidharzsystems kann nur bei detaillierter Kenntnis der StrukturEigenschaftskorrelation erfolgreich vorgenommen werden. Im Folgenden wird beschrieben, wie allein mit Hilfe von thermo-analytischen Methoden ein umfangreiches Bild einer solchen Korrelation gewonnen werden kann.

\section{Experimentelle Untersuchungen}

Zur Charakterisierung von Kunststoffen gibt es eine Reihe von Analysenmethoden, die üblicherweise eine physikalische Messgröße in Abhängigkeit von der Temperatur registrieren. Eine sehr häufig eingesetzte Methode ist die dynamische Differenzkalorimetrie (Differential Scanning Calorimetry, DSC). Sie erlaubt Aussagen über die Glasumwandlungstemperatur $\mathrm{T}_{\mathrm{g}}$, das Schmelz- und Kristallisationsverhalten und den Reaktionszustand (z. B. den Vernetzungsgrad) eines polymerbasierten Systems.

In einer DSC-Apparatur wird mit einem kleinen Ofen Wärme zur Probe und zu einer inerten Referenz übertragen. In unmittelbarem Kontakt zur Probe und Referenz sind Temperatursensoren (Thermoelemente) angebracht. Sie ermöglichen die Registrierung der Temperaturdifferenz zwischen Probe und Referenz während des zeitlinearen Aufheizens. Dieses Signal dient als Maß für den Wärmefluss zwischen Probe und Referenz. Ein exothermer Prozess zeigt ein positives Signal, während endotherme Prozesse negative Peaks hervorrufen. Aus solchen Kurven kann die Enthalpie, d. h. die "Wärmetönung“ einer Umwandlung, berechnet werden. Sie entspricht der integrierten Fläche des zur Umwandlung gehörigen Peaks. Die Glasumwandlung erscheint als Stufe in der Basislinie des registrierten DSC-Signals, da die Probe im Bereich der Glasumwandlung ihre Wärmekapazität ändert. Die Ver-

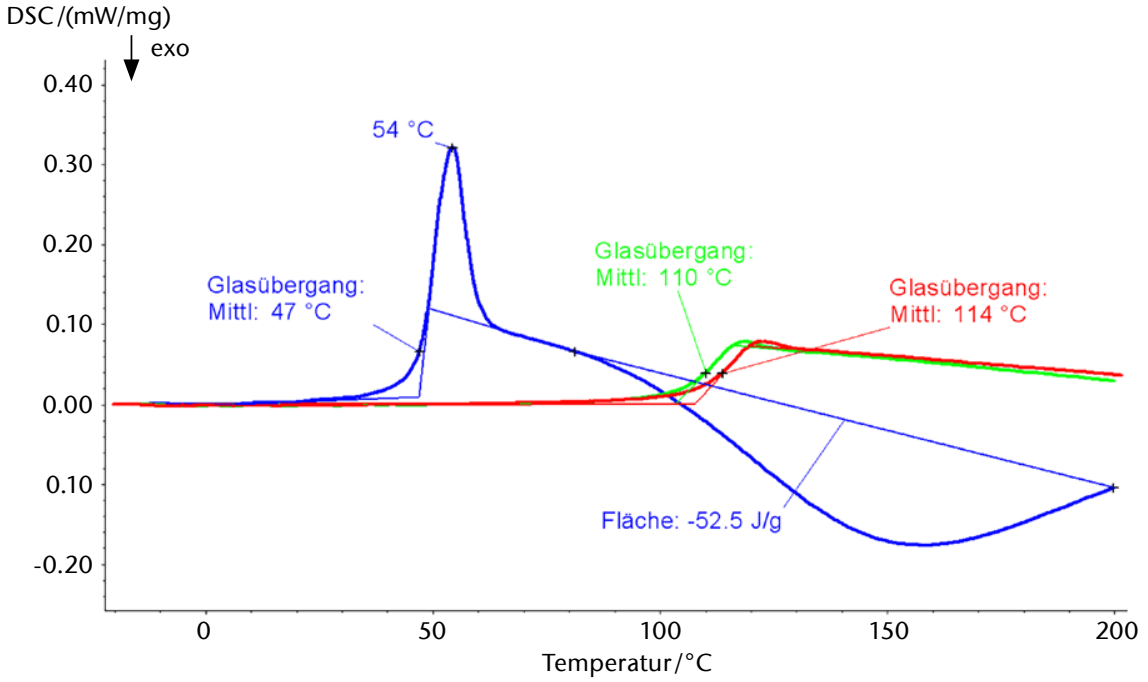

Abb. 5) DSC-Messkurve der Probe DA $1+295$. Blau: 1. Heizen, grün: 2. Heizen, rot: 3. Heizen.

netzung von Kettenmolekülen während des Härtungsprozesses erzeugt als exothermer Vorgang einen Peak in der DSC-Kurve.

Im Folgenden werden Untersuchungen an Epoxidharz-Systemen beschrieben, die eine Struktur-Eigenschaftskorrelation zum Ziel haben. Variiert werden das Harz-Härter-Äquivalentverhältnis, die Zusammensetzung des Harz- und Härter-Systems einschließlich eines reaktiven Verdünners sowie der eingesetzte Katalysator gemäß Tab. 1. Als Eigenschaftskennwerte werden $T_{g}$ des härtenden Systems und die bei der Härtung auftretende Reaktionsenthalpie diskutiert.

Die Bezeichnung in Tab. 1 benennt die bei Raumtemperatur (RT) in Petrischalen gegossenen und gehärteten Proben von ca. $120 \mathrm{~mm}$ Durchmesser und $2 \mathrm{~mm}$ Dicke. Dabei bedeuten:

\begin{tabular}{|c|c|}
\hline Es & Ester-Epoxid \\
\hline Et & Ether-Epoxid \\
\hline $\mathrm{DA}_{1}$ & Diamin-Härter 1 \\
\hline $\mathrm{DA}_{2}$ & Diamin-Härter 2 \\
\hline $\begin{array}{l}\mathrm{DA}_{1+2} \\
\text { Index }\end{array}$ & $\begin{array}{l}\text { Diamingemisch als Härter } \\
\text { Äquivalentverhältnis }\end{array}$ \\
\hline
\end{tabular}

Die DSC-Messungen wurden mit einer DSC 204 Phoenix der Fa. Netzsch Gerätebau $\mathrm{GmbH}$, Selb, mit einer Heizgeschwindigkeit von $10 \mathrm{~K} / \mathrm{min}$ durchgeführt. Die Messproben wurden aus den gehärteten Platten ausgeschnitten und gespalten, sodass jeweils eine konstante Einwaage von ca. $20 \mathrm{mg}$ und eine Dicke von ca. $1 \mathrm{~mm}$ gewährleistet waren.

\section{Ergebnisse und Diskussion}

Aus den gemessenen DSC-Kurven konnten folgende charakteristische Werte für die Epoxidharz-Systeme abgeleitet werden: Glasübergangstemperatur, Temperaturlage des Relaxationspeaks und die Restenthalpie.

Beispielhaft sind in Abb. 5 die DSCMessdaten für die Probe $\mathrm{DA}_{1+2} 95$ grafisch dargestellt.

Besonders auffällig ist der intensive endotherme Peak kurz oberhalb der Reaktionstemperatur im ersten Heizlauf. Die Niedertemperatur-Flanke des Peaks wird anfänglich durch die stufenförmige Erhöhung des DSC-Signals bei Erreichen der Glasübergangstemperatur $T_{g}$ erzeugt. Der endotherme Peak hat seine Ursache in der StrukturRelaxation bei Einsetzen der Segmentbeweglichkeit oberhalb von $\mathrm{T}_{\mathrm{g}}$. Beim erstmaligen Erreichen von etwa 80 ${ }^{\circ} \mathrm{C}$ (nach dem Härten der Probe bei RT) setzt eine weitere Reaktion ein. Bei der Probenherstellung wurde die Reaktion bei RT durch die mangelnde Moleküldynamik abgebrochen, obwohl noch funktionelle Gruppen zur Verfügung standen. Dieser Zustand wird durch ein $\mathrm{T}_{\mathrm{g}}=47^{\circ} \mathrm{C}$ charakterisiert. Durch Einsetzen der Segmentbeweglichkeit oberhalb dieses $\mathrm{T}_{g}$ kann ein weiterer Umsatz erfolgen. Ein Maß für die nun noch ablaufende Reaktion ist die Restenthalpie, wie sie durch die Fläche unter dem DSC-Signal angezeigt wird. Nach Ablauf dieser Reaktion wird ein um mehr als $60 \mathrm{~K}$ höheres $\mathrm{T}_{\mathrm{g}}$ erreicht, wie dem 2. Heizlauf in Abb. 5 zu entnehmen ist. Bei einem 3. Heizen wird schließlich $\mathrm{T}_{\mathrm{g}}$ nur 
Tab.1: Glasübergangstemperatur $\mathrm{T}_{g}$ der drei Heizläufe, Restenthalpie $\Delta \mathrm{H}$ und Maximumlage des endothermen Peaks $\mathrm{T}_{\max }$

\begin{tabular}{|c|c|c|c|c|c|c|c|}
\hline Bezeichnung & \multicolumn{3}{|c|}{$\mathrm{T}_{\mathrm{g}} /{ }^{\circ} \mathrm{C}$} & \multicolumn{2}{|c|}{$\mathrm{T}_{g}(2)-\mathrm{T}_{g}(1), \mathrm{T}_{g}(3)-\mathrm{T}_{g}(1) / \mathrm{K}$} & \multirow{2}{*}{$\begin{array}{c}\Delta \mathrm{H} / \mathrm{J} \cdot \mathrm{g}^{-1} \\
25\end{array}$} & \multirow{2}{*}{$\begin{array}{c}\mathrm{T}_{\max } /{ }^{\circ} \mathrm{C} \\
63\end{array}$} \\
\hline $\mathrm{DA}_{1} 100$ & 58 & 106 & 109 & 48 & 51 & & \\
\hline $\mathrm{DA}_{1+2} 100$ & 58 & 107 & 113 & 49 & 55 & 60 & 63 \\
\hline $\mathrm{DA}_{1+2} 85$ & $54^{1)}$ & 101 & 108 & 47 & 54 & 19 & 62 \\
\hline \multirow[t]{2}{*}{$\mathrm{DA}_{1+2} 8570 G$} & $63 / 77$ & 104 & 108 & $41 / 27$ & $45 / 31$ & 4 & 82 \\
\hline & $63 / 77$ & 102 & 109 & $39 / 25$ & $46 / 32$ & 2 & 83 \\
\hline $\mathrm{DA}_{1+2} 95$ & $47_{50}$ & $110_{109}$ & $114_{103}$ & $63_{59}$ & $67_{63}$ & $52_{40}$ & $54_{56}$ \\
\hline $\mathrm{DA}_{1+2} 90$ & $51_{51}$ & $102_{102}$ & $108_{108}$ & $51_{51}$ & $57_{57}$ & $26_{26}$ & $57_{57}$ \\
\hline $\mathrm{DA}_{1+2} 80$ & 43 & 89 & 97 & 46 & 54 & 21 & 51 \\
\hline $\mathrm{DA}_{1} 95$ & 47 & 107 & 111 & 60 & 64 & 42 & 54 \\
\hline $\mathrm{DA}_{1} 90$ & 47 & 99 & 105 & 52 & 58 & 23 & 54 \\
\hline $\mathrm{DA}_{1} 80$ & 44 & 84 & 92 & 40 & 48 & 7 & 51 \\
\hline DA,95 & 50 & 105 & 111 & 55 & 61 & 29 & 56 \\
\hline DA, 90-90/10 & 57 & 110 & 112 & 53 & 55 & 32 & 62 \\
\hline $\mathrm{DA}_{1}, 90-80 / 20$ & 44 & 75 & 81 & 31 & 37 & 1 & 51 \\
\hline $\mathrm{DA}_{1} 80-90 / 10$ & 39 & 70 & 78 & 31 & 39 & 2 & 48 \\
\hline $\mathrm{DA}_{1} 90-95 / 5$ & 50 & 97 & 102 & 47 & 52 & 16 & 56 \\
\hline $\mathrm{DA}_{1} 90+10$ & $49_{54}$ & $99_{103}$ & $100_{103}$ & $50_{49}$ & $51_{49}$ & $84_{83}$ & $56_{60}$ \\
\hline $\mathrm{DA}_{1} 100 / 90-10$ & 49 & 86 & 88 & 37 & 39 & 11 & 56 \\
\hline
\end{tabular}

1) abweichende Dicke des Probekörpers

Tiefgestellte Werte bezeichnen Wiederholungsmessungen mit neuen Probekörpern

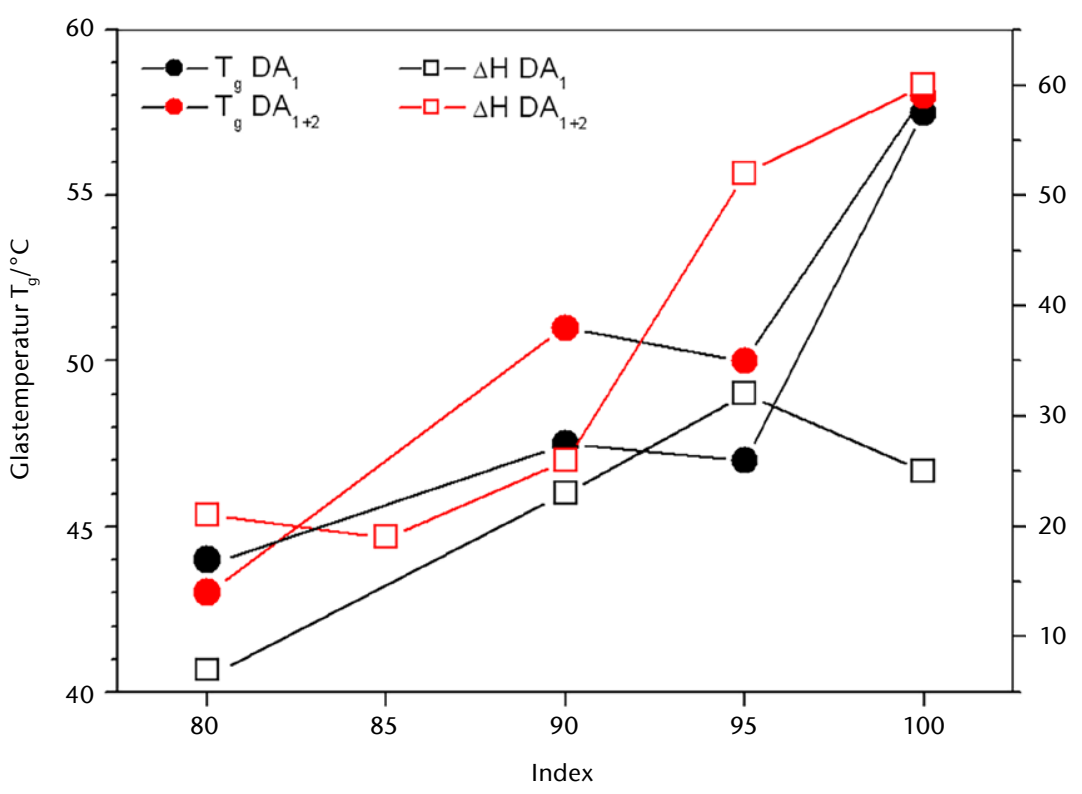

Abb. 6) Glastemperatur $T_{g}$ und Enthalpie $\Delta H$ in Abhängigkeit vom Härteranteil. noch um $4 \mathrm{~K}$ erhöht. Der Umsatz hat nun unter den Messbedingungen sein Maximum erreicht. Die den Messkurven entnommenen Werte sind in Tab. 2 zusammengestellt.

Bei der ersten Betrachtung der Ergebnisse nach Tab. 2 fällt auf, dass durch die Rezepturvariation die größten Unterschiede bei $\Delta \mathrm{H}$ erzielt werden.

In der Abb. 6 sind die Werte für $\mathrm{T}_{\mathrm{g}}$ und $\Delta \mathrm{H}$ in Abhängigkeit vom Index für die beiden Härter $\mathrm{DA}_{1}$ und $\mathrm{DA}_{1+2}$ aufgetragen. In der Tendenz sinkt wie erwartet $\mathrm{T}_{\mathrm{g}}$ mit dem geringeren Einsatz von Härter, da die erzielte Vernetzungsdichte mit geringerem Anteil an Härter ebenfalls sinkt (es sind weniger Verknüpfungsmöglichkeiten vorhanden). Die Abhängigkeit ist in erster Näherung etwa gleichlaufend. Bei der ermittelten Restenthalpie dagegen treten bei höheren Indices deutliche Abweichungen im Verhalten der Systeme mit Härter DA 
und $\mathrm{DA}_{1+2}$ auf. Mit DA lässt sich unter den gegebenen Reaktionsbedingungen ein höherer Umsatz erzielen. Die Restenthalpie ist beim $\mathrm{DA}_{1}$ etwa 3-fach niedriger als beim $\mathrm{DA}_{1+2}$ (bei einem Index von 100). $T_{g}$ dagegen ist bei beiden Systemen etwa gleich, und die Differenz zwischen den Werten bei einem Index von 80 bzw. 100 beträgt lediglich $10 \mathrm{~K}$. Diese Unterschiede lassen sich mit der chemischen Struktur korrelieren. Beim Einsatz einer Mischung von DA, und $\mathrm{DA}_{1+2}$ bei Probe DA $90+10$ wird die Reaktivität ganz besonders stark beeinflusst. Hier erreicht $\Delta \mathrm{H}$ einen Wert von über 80 J/g; der Wert für Tg dagegen ordnet sich mit ca. $50{ }^{\circ} \mathrm{C}$ auf einem durchschnittlichen Niveau ein.

Bei einem Gemisch aus Ester und Ether wie bei den Proben DA,90-80/20 und $\mathrm{DA}_{1} 80-90 / 10$ (Index 90 bzw. 80) wird ein fast vollständiger Umsatz erreicht, während $T_{g}$ besonders niedrig bleibt. Für eine ausführliche Diskussion müssen noch zusätzliche strukturelle Parameter herangezogen werden (das gilt auch für die Ergebnisse an den restlichen Proben).

Am Beispiel der Probe $\mathrm{DA}_{1+2} 8570 \mathrm{G}$ kann der Einfluss der Reaktionstemperatur demonstriert werden.

Die Messkurve gemäß Abb. 7 zeigt beim 1. Heizen zwei Glasübergangsstufen. Die erste ist wie erwartet gegenüber den anderen bei Raumtemperatur gehärteten Proben um etwa $10 \mathrm{~K}$ erhöht. Die Reaktion ist aber unter den gegebenen Bedingungen noch nicht abgeschlossen. Bei Überschreiten des erreichten $\mathrm{T}_{\mathrm{g}}$ beginnt die Nachreaktion, die dann mit Erreichen des neuen $\mathrm{T}_{g}$ bei annähernd der Reaktionstemperatur wieder „einfriert“.

Mit weiterer Temperaturerhöhung erfolgt der maximal mögliche Umsatz die Restenthalpie geht gegen null. Nach einer Temperaturbeanspruchung bis $200{ }^{\circ} \mathrm{C}$ (während der Messung) wird eine $T_{g}$ von $102{ }^{\circ} \mathrm{C}$ erreicht. Bei einem weiteren Lauf bis $200^{\circ} \mathrm{C}$ wird $\mathrm{T}_{\mathrm{g}}$ schließlich noch einmal um $7 \mathrm{~K}$ erhöht. Auch diese Messung zeigt, dass der erzielbare Wert für $\mathrm{T}_{\mathrm{g}}$ nicht wesentlich höher als die Härtungstemperatur werden kann.

\section{Schlussfolgerungen}

Es wird bestätigt, dass die DSC gut geeignet ist, ein umfangreiches Bild von reagierenden Polymersystemen

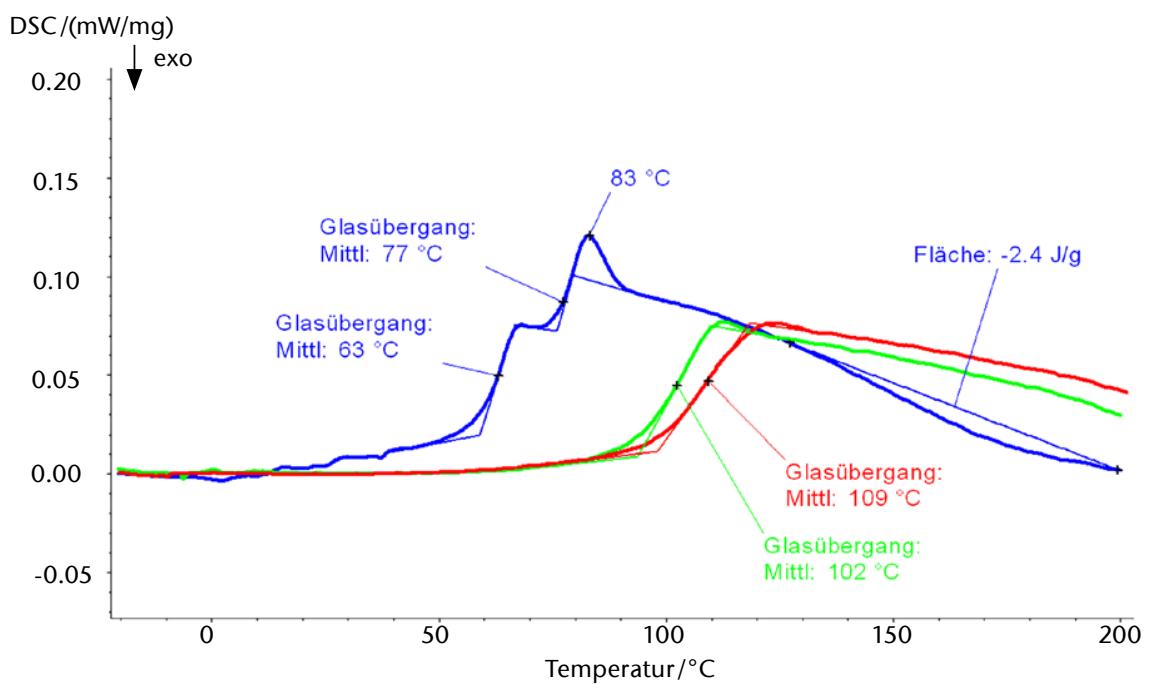

Abb. 7) DSC-Kurven der bei $70^{\circ} \mathrm{C}$ gehärteten Probe $D A_{1+2} 8570 \mathrm{G}$

zu liefern. Wie erwartet ist dieses Bild nicht vollständig. Es bedarf der Anwendung weiterer Messmethoden zur genaueren Charakterisierung auf molekularer Ebene.

Die Messungen an dem reaktiven Epoxidharz-System belegen die Tatsache, dass sich ein polymeres Netzwerk nur bis zum Erreichen von $\mathrm{T}_{\mathrm{g}}$ verfeinern lässt. Mit Erreichen von $\mathrm{T}_{g}^{g}$ wird die molekulare Beweglichkeit so stark eingeschränkt, dass eine weitere Vernetzung nicht mehr erfolgen kann. Dazu bedarf es einer Erhöhung der Temperatur oder der Weichmachung durch flexible bzw. niedermolekulare Substanzen, die nach der Härtung wieder entfernt werden oder als Weichmacher im Harz verbleiben. Das Probensystem veranschaulicht das Zusammenspiel von molekularer Beweglichkeit und thermisch aktivierter Reaktivität. Für eine detaillierte Beschreibung des Epoxidharz-Systems bedarf es einer weiteren systematischen Variation der Zusammensetzung und des Einsatzes weiterer Charakterisierungsmethoden. Wichtig für die Ableitung der Gebrauchseigenschaften und der Stabilität sind zusätzliche Informationen zur Kinetik der Vernetzungsreaktion. Erste Messungen für entsprechende Untersuchungen wurden bereits durchgeführt.

Allein aus den thermo-analytischen Messdaten lassen sich wichtige Hinweise auf die optimierte Einstellung von Systemparametern ableiten. So zeigen die Härter-Kombinationen be- sonderes Potenzial für optimale Formulierungen. Mit den abgeleiteten Erkenntnissen zur molekularen Struktur der Netzwerke ergeben sich zusätzliche Möglichkeiten bei der weiteren Vorhersage der Langzeitstabilität dieser Systeme.

Das entwickelte Epoxidharzsystem gestattet die nachträgliche Ausstattung von Fußbodenheizungsrohren mit einer praktisch sauerstoffundurchlässigen Schutzschicht in einer bestehenden Heizungsanlage. Die Sauerstoffbarrierewirkung übertrifft die nach DIN 4726 geforderten Werte um mehr als eine Größenordnung. (gefordert: $\leq 0,32 \mathrm{mg} / \mathrm{m}^{2} \cdot \mathrm{d}$, gemessen: $0,02 \mathrm{mg} / \mathrm{m}^{2} \cdot \mathrm{d}$ ).

\section{LITERATUR}

Franck A, Biederbick K (1990) Kunststoff-Kompendium, 3rd edn. Vogel, Würzburg. ISBN: 978-3-8023-0135-3

Gugumus F (1996a) Thermooxidative degradation of polyolefins in the solid state. Part 1. Experimental kinetics of functional group formation. Polym Degrad Stabi 52(2):131-144. doi: 10.1016/0141-3910(95)00229-4

Gugumus F (1996b) Thermooxidative degradation of polyolefins in the solid state. Part 2. Homogeneous and heterogeneous aspects of thermal oxidation. Polym Degrad Stabil 52(2):145-157. doi: 10.1016/01413910(95)00227-8

Gugumus F (1996c) Thermooxidative degradation of polyolefins in the solid state. Part 3. Heterogeneous oxidation model. Polym Degrad Stabil 52(2):159-170. doi 10.1016/0141-3910(95)00228-6

Kriston I (2010) Some aspects of the degradation and stabilization of Phillips type Polyethylene. Ph. D. Thesis, Budapest University of Technology and Economics, Laboratory of Plastics and Rubber Technology

Langer B, Berthold A, Grellmann W, Enderle HF (2012) Mechanische Kurzzeitprüfung zur Bewertung des Verhaltens von PE-Rohrwerkstoffen beim langsamen Risswachstum. Mater Test 54(9):578-583. doi: 10.3139/120.110364 
Mitroka SM, Smiley TD, Tanko JM, Dietrich AM (2013) Reaction mechanism for oxidation and degradation of high density polyethylene in chlorinated water. Polym Degrad Stabil 98(7):1369-1377. doi: 10.1016/j.polymdegradstab.2013.03.020

Ojeda T, Freitas A, Birck K, Dalmolin E, Jacques R, Bento F, Camargo F (2011) Degradability of linear polyolefins un der natural weathering. Polym Degrad Stabil 96(4):703707. doi: 10.1016/j.polymdegradstab.2010.12.004

Prüfbericht (2014) K 14 1228.1, Sauerstoffdichtheitsprüfung nach DIN 4726. Technische Universität Darmstadt, Zentrum für Konstruktionswerkstoffe

Robertson D (2013) The oxidative resistance of polymeric geosynthetic barriers (GBR-P) used for road and railway tunnels. Polym Test 32(8):1594-1602. doi: 10.1016/j.po lymertesting.2013.09.012

Schröder HF, Munz M, Böhning M (2008) A New Method for Testing and Evaluating the Long-Time Resistance to Oxidation of Polyolefinic Products. Polym Polym Compos 16(1):71-79

Sittiho M (2011) Quantitative Beurteilung des Gaseintrages in thermische Energieversorgungssysteme aufgrund
der Gaspermeation. Dissertation, Technische Universität der Gaspermeation. Dissertation, Tech
Chemnitz, Fakultät für Maschinenbau

Viebke J, Gedde UW (1997) Antioxidant diffusion in polyethylene hot-water pipes. Polym Eng Sci 37(5):896-911. doi: 10.1002/pen.11733

Vogt H, Enderle HF, Schulte U, Hessel J (2008) Thermal ageing of $P E 100$ pipes for accelerated lifetime prediction under service. Plastic Pipes XIV, Budapest

Weltschev M (2010) Vergleich der Materialkennwerte von Formstoffen aus Polyethylen hoher Dichte mi dem Baumusterverhalten von Gefahrgutverpackungen BAM-Dissertationsreihe, vol 53. Bundesanstalt für Materialforschung und -prüfung. ISBN: 978-3-9812910-9-4

Whelton AJ, Dietrich AM (2009) Critical considerations for the accelerated ageing of high-density polyethylene potable water materials. Polym Degrad Stabil 94(7):1163-1175. doi: 10.1016/j.polymdegradstab.2009.03.013

\section{AUTOREN}

Dipl.-Chem. Eckhart Kornejew

Dr. rer. nat. Harald Goering

Prof. Dr. rer. nat. Michael Herzo

iMEP - Institut für Material, Entwicklung und Produktion

E-Mail für Korrespondenz:

michael.herzog@th-wildau.de 